\title{
A Rapid Life Cycle Assessment Method based on Green Features in Supporting Conceptual Design
}

\author{
Qiang Meng', Fang-yi Li',", Li-rong Zhou', Jing Li', Qin-qin Ji', and Xiaodong Yang ${ }^{2}$ \\ 1 Key Laboratory of High Efficiency and Clean Mechanical Manufacture Ministry of Education, School of Mechanical Engineering, Shandong University, Jinan, 250061, China \\ 2 Inspur Electronic Information Industry Co., Ltd., Jinan, 250014, China \\ \# Corresponding Author / E-mail: lifangyi@sdu.edu.cn, TEL: +86-0531-88392003, FAX: +86-0531-88574135
}

KEYWORDS: Rapid life cycle assessment, Green features, Conceptual design, Uncertainty process, Intuitionistic fuzzy, Monte carlo

\begin{abstract}
A Rapid Life Cycle Assessment (RLCA) method based on Green features is proposed in order to solve the inherent limitations of conventional Life Cycle Assessment (LCA), such as long period, massive data requirement, which result in difficulties in supporting product conceptual design. Firstly, Green Feature is proposed in supporting the LCA in conceptual design, where a mapping relationship is established between green feature and design information to achieve the transformation from the design information to green features. Secondly, product conceptual design model is proposed based on modular configuration. The approximate products program will be obtained through retrieval and matching of each module of product using the knowledge base and case base. Thirdly, Intuitionistic Fuzzy theory and Monte Carlo method are respectively used to process the qualitative and quantitative uncertain information of green features in order to ensure the accuracy of the evaluation results. Besides, the appropriate life cycle impact assessment method is selected to complete the life cycle impact assessment and obtain the LCA results. Consequently, the RLCA of product design program is completed to support product green design. Finally, a fan of a ventilation system is studied as an example to verify the proposed RLCA theory.
\end{abstract}

Manuscript received: June 18, 2014 / Revised: December 10, 2014 / Accepted: December 15, 2014

\section{NOMENCLATURE}

LCA $=$ Life Cycle Assessment

RLCA $=$ Rapid Life Cycle Assessment

LCIA $=$ Life Cycle Impact Assessment

$\mathrm{GF}=$ Green Features

\section{Introduction}

Green design and manufacturing has become a hot research point, and life cycle assessment has become an important tool to support product green design. But according to the steps of the conventional life cycle assessment, a large amount of time and energy would be spent on collecting and processing the data and the accuracy of the data will affect the reliability of the evaluation results to a great extent. The idea of simplified life cycle assessment has been proposed by organizations and scholars in order to solve the inherent limitations of conventional Life Cycle Assessment (LCA) such as high cost, long period, massive data requirement and high requirement of data accuracy.

The report from SETAC put forward the thought and methods of the simplified life cycle assessment. Zhang Xiaolu etc. carried out an inventory analysis of others' research results and statistical data of related departments and processed the inventory data by using a simplified unification method, then finally conducted the life cycle impact assessment with air conditioning. ${ }^{2}$ J. Y. Ryu etc. analyzed 11 kinds of simplified methods of life cycle assessment in terms of life cycle stages and data acquisition. ${ }^{3}$ Greadel etc. proposed a semiquantitative weighted matrix, namely the ERPA matrix, and analyzed different electronic products. ${ }^{4}$ Hur T etc. put forward a simplified life cycle assessment method based on the matrix method, in order to rapidly identify and screen factors which have great influence on production environment. ${ }^{5}$ Gao Yang etc. used a simplified LCA method to evaluate the green nature of consumable products. ${ }^{6}$ Hyunseop Lee etc. proposed a mathematical model-based evaluation method to determine the environmental burden of the Chemical mechanical planarization process, which may contribute to the greening of the semiconductor process. ${ }^{7} \mathrm{Ju}$ Yeon Lee suggested a simulation-based analysis system for 
sustainable manufacturing, which can assess the impact of manufacturing systems and minimize the negative impact on the environment. ${ }^{8}$ Raoudha Gaha propose a methodology based on feature technology and on the integrations realized on CAD systems to generate alternative scenarios and calculated the environmental impact with CML method.' Abovementioned methods mean to implement the life cycle assessment by using existing life cycle data and tool, through simplifying life cycle phase, the type of environmental impact or data on the basis of the current technical framework or model. Although such methods reduce the appraisal cost and shorten the evaluation time, it would at the same time reduce the accuracy and reliability of life cycle assessment.

In the aspect of the uncertain information processing, Mo Hua etc. proposed a quality evaluation system based on the quality index method and Monte Carlo method..$^{10}$ Lloyd S M etc. thought that Monte Carlo method and Fuzzy Theory can be used to deal with quantitative uncertain information to support Quantitative Decision Making, but pay less attention to application research of qualitative uncertain information. ${ }^{11}$ Benetto E etc. proposed an improved NAIADE method based on the fuzzy technique, and the uncertainty of LCA thus can be comprehensively analyzed. ${ }^{12}$ Lo S. C. etc. proposed an uncertain analysis method which combines Bayes with Monte Carlo, in order to reduce the uncertainty in the process of LCA. ${ }^{13}$ Wang etc. researched by using of multiple regression analysis on the uncertainty arising from solution selection. ${ }^{14}$ Zhu Lihong proposed a comprehensive analysis method which combines qualitative and quantitative methods to solve the uncertainty analysis of LCI. ${ }^{15}$ Ming-Chuan Chiu proposed a method based on fuzzy set theory to tackle the uncertainty of supply chain and highlight the sustainable design concepts considering product disassemblability, closed loop supply chain performance, and carbon footprint. ${ }^{16}$ The scholars mentioned above lucubrated the quality, uncertainty of the LCI data and the approaches to reduce the uncertainty, but the study in terms of how to quantify uncertain information is rare.

A Rapid Life Cycle Assessment (RLCA) method for conceptual design based on Green Features is proposed in this paper. Firstly, Green Feature is proposed in supporting the life cycle assessment in conceptual design stage. Then, the conceptual design model based on modular configuration and green feature exaction model are established to quickly obtain the information needed in LCA. And the Intuitionistic Fuzzy theory and Monte Carlo method are respectively used to process the qualitative and quantitative uncertain information of green features in order to ensure the accuracy of the evaluation results.

\section{Green Feature}

Conventional design mainly considers the achievement of product functional structure, feasibility of the process and economy etc., which are shown as materials choosing, processing method and processing parameters in the product design. ${ }^{17}$ The green design pays more attention to the environmental performance such as consumptions, emissions, noise and recycling etc. without compromising product performance and increasing the cost as much as possible. Green Feature is introduced in this paper, which is a mapping between conventional design variables and green attributes. Thereby, green design information of the product can be extracted and filtered from conventional information.

Based on set theory, Green Feature (GF) is a collection of green information within relevant product life cycle, which can be described as Eq. (1):

$$
G F=F_{T} \cdot F_{I} \cdot F_{G}
$$

Where

$F_{T}=\left\{T_{i}, i=1\right.$ to $n \mid$ Raw material acquisition, Manufacturing, Using, End of life dispose $\left.\cdots\right\}$

$F_{I}=\left\{I_{j}, j=1\right.$ to $m \mid$ Component and part 1, Component and part 2, Component part 3, $\left.\cdots\right\}$

$F_{G}=\left\{G_{k}, k=1\right.$ to $p \mid$ Material feature, Energy feature, Emission feature, $\left.\cdots\right\}$

A conclusion can be drawn from analyzing Eq. (1) that green feature includes three levels of information: from the aspect of time domain, green feature covers the whole process of Life Cycle from raw material acquisition, manufacturing, transportation, using to end of life dispose; from the aspect of physics domain, green feature involves each part and component of the product; from the aspect of relation domain, green feature realizes the transfer of project from conventional design domain to green design domain.

Green feature is a representation of product design information which presents general design information mapping to the green feature according to certain rules. Because green features pay more attention to product greenness-related information, the representation of product design program based on green features can reflect the greenness more intuitively, which facilitate the evaluation of program greenness.

\section{The Rapid Life Cycle Assessment (RLCA) Model}

As the global environment problems are increasingly prominent, more and more designers begin to pay attention to green design in order to pursue the sustainable development of product. As the key stage of product green design, conceptual design has a decisive influence on the structural performance, environmental performance and cost of product. However, challenges exist therein such as less restrain on designers, uncertainty of design information and difficulties in acquiring related green information. This paper firstly establishes the product conceptual design model based on modular product structure configuration. Product conceptual design will be done from the perspective of the whole life cycle and modular design, and the approximate products program and related design information will be obtained through retrieval and matching of each module of product using the knowledge base and case base. Secondly, a mapping model between design information and green features is established to achieve the transformation from design information to green feature. Finally, in order to ensure the accuracy of the evaluation results, the uncertain information in the green feature inventory is disposed and then the rapid life cycle assessment of product program is conducted. As shown in Fig. 1:

\section{Product Conceptual Design Model based on Modular Configuration}

Firstly, the life-cycle modular design approach is used in product conceptual design. Then, functional module is matched with the 


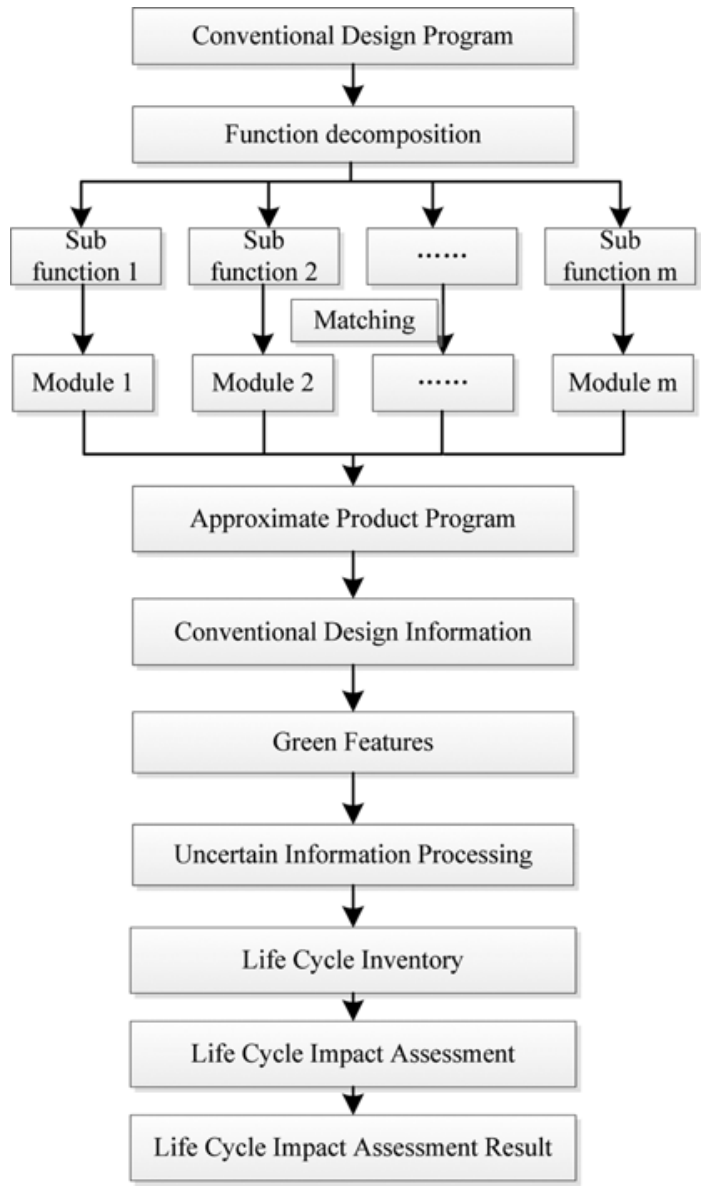

Fig. 1 Rapid life cycle assessment model

functional characteristics through calculating the similarity in the module library to quickly complete the product approximate design program. This will not only avoid the issues such as less information, high uncertainty etc. in conceptual design stage, but also can get a lot of design information needed for life cycle assessment without detailed design, which greatly improves the design efficiency and saves time and costs.

\subsection{Matter-Element Representation of Function Module}

In this paper, Matter-element theory is used to describe the function of the module for calculating similarity. Element was usually expressed as $R=(N, C, v)$. Here, $N, C$ and $v$ respectively represent the matter, features and value.

If the program a function has $n$ characteristic indexes and $m$ corresponding similar modules, the complex matter-element can be expressed as Eq. (2) based on matter-element theory:

$$
R_{m n}=\left[\begin{array}{cccccc} 
& M_{0} & M_{1} & M_{2} & \ldots & M_{m} \\
C_{1} & v_{10} & v_{11} & v_{12} & \ldots & v_{1 m} \\
C_{2} & v_{20} & v_{21} & v_{22} & \ldots & v_{2 m} \\
\ldots & \ldots & \ldots & \ldots & \ldots & \ldots \\
C_{n} & v_{n 0} & v_{n 1} & v_{n 2} & \ldots & v_{n m}
\end{array}\right]
$$

In the above Equation,

$C_{i}(i=1,2, \ldots, n)$ represents the $\mathrm{i}$-th characteristic of function module;
$M_{j}(j=0,1,2, \ldots, m)$ represents the $\mathrm{j}$-th similar module , M0 refers to the designer's ideal function module;

$v_{i j}(i=1,2, \ldots, n ; j=0,1,2, \ldots, m)$ represents the $\mathrm{i}$-th eigenvalue of $\mathrm{j}$-th module, M0 represents the i-th eigenvalue of designer ideal function module.

The characteristic index has qualitative and quantitative two forms. $v_{i j}(i=1,2, \ldots, n ; j=0,1,2, \ldots, m)$ represents the value of quantitative characteristics and the membership according to the fuzzy mathematical theory.

\subsection{Similarity Calculation}

Currently, common methods for calculating similarity are divided into the distance methods and cosine methods. And the more each score tends to proportional, regardless of the numerical differences, the greater the degree of similarity in cosine methods. Therefore, an improved Euclidean distance method is used in this paper. However, the Euclidean distance method requires that dimension of all data must be uniform, so the composite element must be normalized before similarity calculation, which shown as follows:

$$
R_{m n}^{\prime}=\left[\begin{array}{cccccc}
M_{0} & M_{1} & M_{2} & \ldots & M_{m} \\
C_{1} & x_{10}^{\prime} & x_{11}^{\prime} & x_{12}^{\prime} & \ldots & x_{1 m}^{\prime} \\
C_{2} & x_{20}^{\prime} & x_{21}^{\prime} & x_{22}^{\prime} & \ldots & x_{2 m}^{\prime} \\
\ldots & \ldots & \ldots & \ldots & \ldots & \ldots \\
C_{n} & x_{n 0}^{\prime} & x^{\prime}{ }_{n 1} & x_{n 2}^{\prime} & \ldots & x_{n m}^{\prime}
\end{array}\right]
$$

Where, When it is qualitative feature, it needs no further standardization, $x_{i j}^{\prime}=x_{i j}$ and $x_{i j} \in[0,1]$;

When it is quantitative characteristic feature:

$$
x^{\prime}{ }_{i j}=x_{i j} / \sqrt{\sum_{j=0}^{m} x_{i j}^{2}},(i=1,2, \ldots, n ; j=0,1,2, \ldots, m)
$$

Similarity calculation of each module to the ideal module:

$$
S_{j}=\sum_{i=1}^{n} w_{i} \sqrt{\left(x_{i j}^{\prime}-x_{i 0}^{\prime}\right)^{2}},(i=1,2, \ldots, n ; j=0,1,2, \ldots, m)
$$

$w_{i}(i=1,2, \ldots, n)$ represents the weight value of the i-th feature in the composite element, where $\sum_{i=1}^{n} w_{i}=1$.

If $S_{p}=\min \left(S_{1}, S_{2}, S_{3}, \ldots, S_{m}\right)$, the p-th module has the highest similarity to the reference module which can be regarded as the optimal solution. Similarly, other modules can be matched out in order, which constitute the approximation program.

\section{Extraction of Green Feature}

Modular product is a collection of functional modules, so functional module should be considered as the unit when extracting Green Feature. Based on the idea of product modular design, design information of each functional module is firstly expressed mathematically with a matrix (IT) so as to used in the following calculation; then the module IT matrix is summarized to obtain the product IT matrix; and finally product green features are extracted by 


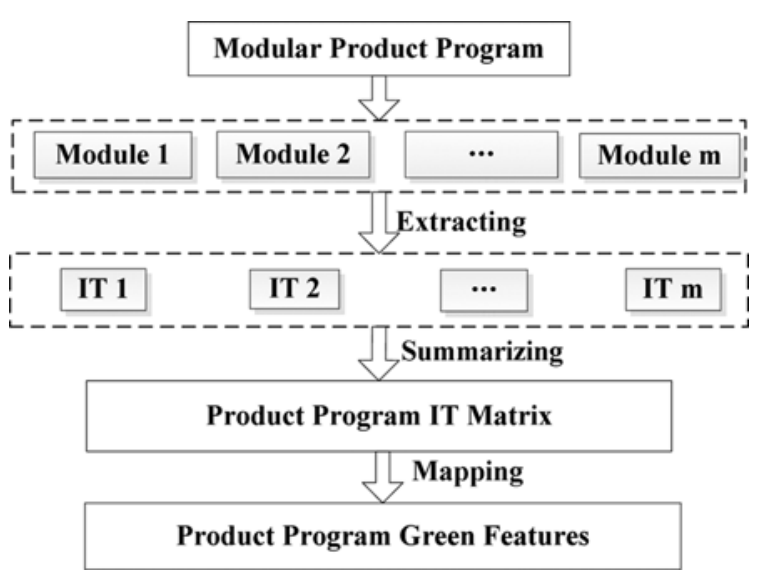

Fig. 2 Green feature extraction processes of modular product

mapping matrix, as shown in Fig. 2.

The design information Matrix IT presents the conventional design information of each module of product program in accordance with the life cycle stages such as raw material acquisition, manufacturing, storage, transportation, maintenance, scrap disposal and so on in the form of generalized matrix multiplication, as shown in Eq. (6).

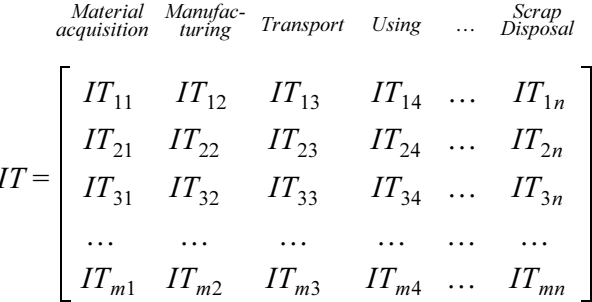

After obtaining product IT Matrix, a green feature mapping matrix FG is built to extract and filter green feature information for evaluation process from conventional design information based on the mapping relationship between green features and product design parameters, as shown in Eq. (7):

$$
F_{G}=\left[\begin{array}{cccc}
F_{1}() & F_{2}() & \ldots & F_{k}() \\
F_{1}() & F_{2}() & \ldots & F_{k}() \\
\ldots & \ldots & \ldots & \ldots \\
F_{1}() & F_{2}() & \ldots & F_{k}()
\end{array}\right]
$$

The green features related to environment impact such as scarcity, degradability, radioactivity, reproducibility, recyclability, and energy consumption, emissions etc. are filtered out from the product information and classified through above function. Thereby, the green feature matrix GF is obtained, as shown in Eq. (8).

$$
G F=I T \cdot F_{G}=\left[\begin{array}{cccc}
I T_{11} & I T_{12} & \ldots I T_{1 n} \\
I T_{21} & I T_{22} & \ldots I T_{2 n} \\
\ldots & \ldots & \ldots & \ldots \\
I T_{m 1} & I T_{m 2} & \ldots & I T_{m n}
\end{array}\right]\left[\begin{array}{cccc}
F_{1}() & F_{2}() & \ldots & F_{k}() \\
F_{1}() & F_{2}() & \ldots & F_{k}() \\
\ldots & \ldots & \ldots & \ldots \\
F_{1}() & F_{2}() & \ldots & F_{k}()
\end{array}\right]
$$

$$
=\left[\begin{array}{cccc}
\sum_{t=1}^{n} F_{1}\left(I T_{1 t}\right) & \sum_{t=1}^{n} F_{2}\left(I T_{1 t}\right) & \ldots & \sum_{t=1}^{n} F_{k}\left(I T_{1 t}\right) \\
\sum_{t=1}^{n} F_{1}\left(I T_{2 t}\right) & \sum_{t=1}^{n} F_{2}\left(I T_{2 t}\right) & \ldots & \sum_{t=1}^{n} F_{k}\left(I T_{2 t}\right) \\
\ldots & \ldots & \ldots & \ldots \\
\sum_{t=1}^{n} F_{1}\left(I T_{m t}\right) & \sum_{t=1}^{n} F_{2}\left(I T_{m t}\right) & \ldots & \sum_{t=1}^{n} F_{k}\left(I T_{m t}\right)
\end{array}\right]
$$

Here matrix form is used to express design information, and matrix multiplication form is used to extract and express green information instead of the mathematical matrix multiplication.

Generally, the product program green features include all the green information of product in the whole life cycle. Therefore, polymerizing the congeneric green features in Eq. (8) can obtain the green features of the product program, as shown in Eq. (9):

$G F_{k}=\sum_{t=1}^{n} F_{k}\left(I T_{1 t}\right)+\sum_{t=1}^{n} F_{k}\left(I T_{2 t}\right)+\ldots+\sum_{t=1}^{n} F_{k}\left(I T_{m t}\right)=\sum_{h=1}^{m} \sum_{t=1}^{n} F_{k}\left(I T_{h t}\right)(9)$

Finally, the green features of the product program can be expressed as shown in Eq. (10):

$$
G F=\left(\begin{array}{llll}
G F_{1} & G F_{2} & \ldots & G F_{k}
\end{array}\right)
$$

The properties of green features fully considered concern of different populations when defined, so analyzing the properties of product green features can obtain the information relevant to evaluation. Thereby, the LCI and LCIA of product program can be realized in the following.

\section{Processing of Uncertain Information}

The green feature of design project is extracted and filtered through green feature mapping model, which can be divided into qualitative features and quantitative features. For uncertain green features, Intuitionistic Fuzzy Numbers of Fuzzy theory is used to study the qualitative features, while the Monte Carlo method is used to conduct simulated analysis of quantitative features, regarding the variance of which as uncertainty factor in uncertainty analysis. The technology roadmap of uncertain information processing is presented as in Fig. 3.

\subsection{Processing of Qualitative Feature}

Some certain green features (such as material scarcity, removability, etc.) cannot be specifically quantified, thus some fuzzy numbers and vague language are needed in evaluation, such as "very good", "very bad", "relatively small", "relatively large" and so on. To a certain degree, intuitionistic fuzzy number is a proper tool to process and quantify the fuzzy evaluation, which can convert linguistic variables into intuitionistic fuzzy numbers in Table $1 .{ }^{18}$ For qualitative uncertain green feature, during the polymerization process of green features, the subfeatures can be presented by fuzzy numbers, which are then weighted to obtain the integrated fuzzy number, namely the value of the green feature. 


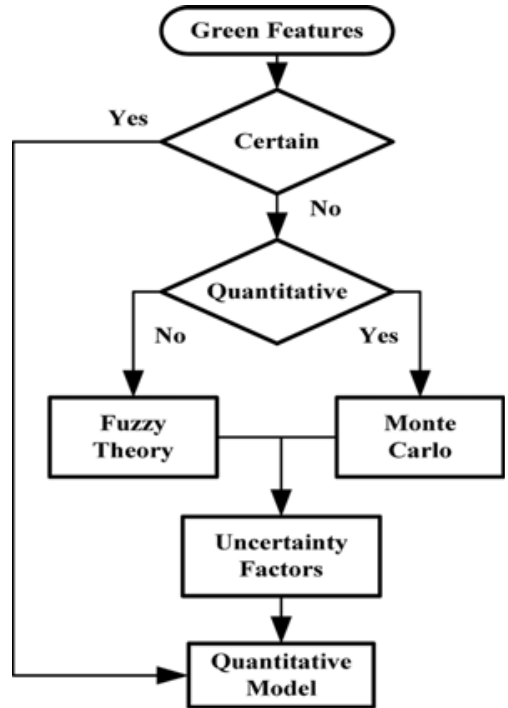

Fig. 3 Uncertain information processing technology roadmap

Table 1 Linguistic variables and intuitionistic fuzzy numbers in correspondence

\begin{tabular}{cc}
\hline Linguistic variable & Intuitionistic fuzzy value \\
\hline Extreme poor & $(0.05,0.95,0.00)$ \\
\hline Very poor & $(0.15,0.80,0.05)$ \\
\hline Poor & $(0.25,0.65,0.10)$ \\
\hline Medium poor & $(0.35,0.55,0.10)$ \\
\hline Medium & $(0.50,0.40,0.10)$ \\
\hline Medium good & $(0.65,0.25,0.10)$ \\
\hline Good & $(0.75,0.15,0.10)$ \\
\hline Very good & $(0.85,0.10,0.05)$ \\
\hline
\end{tabular}

For convenience, this thesis presents relevant concepts and operational laws of intuitionistic fuzzy numbers on the basis of research in. ${ }^{19-21}$

The intuitionistic fuzzy set:

Let $\mathrm{X}=\{\mathrm{x} 1, \mathrm{x} 2, \ldots \ldots, \mathrm{xn}\}$ be a nonempty set, then

$$
A=\left\{\left[x, \mu_{A}(x), v_{A}(x)\right] \mid x \in X\right\}
$$

is called intuitionistic fuzzy set, among which $\mu_{A}(\mathrm{x})$ and $v_{A}(\mathrm{x})$ represent respectively the membership degree and non-membership degree of the element $\mathrm{X}$ in $\mathrm{X}$ to $\mathrm{A}$, namely $\mu_{A}: \mathrm{X} \rightarrow[0,1], v_{A}$ : $\mathrm{X} \rightarrow[0,1]$, and $0 \leq \mu_{A}(\mathrm{x})+v_{A}(\mathrm{x}) \leq 1, \forall \mathrm{x} \in \mathrm{X}$. For convenience, we refer $\alpha=\left(\mu_{\alpha}, v_{\alpha}\right)$ as intuitionistic fuzzy numbers. In addition,

$$
\pi_{A}(x)=1-\mu_{A}(x)-v_{A}(x), \forall \mathrm{x} \in \mathrm{X}
$$

$\pi_{A}$ represents the uncertainty of element $\mathrm{x}$ in $\mathrm{X}$ belonging to $\mathrm{A}$.

The algorithm of intuitionistic fuzzy numbers:

$$
\begin{gathered}
\alpha 1 \oplus \alpha 2=\left(\mu_{\alpha 1}+\mu_{\alpha 2}-\mu_{\alpha 1} \mu_{\alpha 2}, v_{\alpha 1} v_{\alpha 2}\right) \\
\lambda \alpha=\left[1-\left(1-\mu_{\alpha}\right)^{\lambda}, v_{\alpha}^{\lambda}\right], \lambda>0
\end{gathered}
$$

Intuitionistic fuzzy weighted average operator:
Table 2 The feature value and importance of each subfeature

\begin{tabular}{ccc}
\hline Features & Features value & Importance degree \\
\hline$G F_{i 1}$ & Good & Medium \\
\hline$G F_{i 2}$ & Very good & Very important \\
\hline$G F_{i 3}$ & Very good & Medium \\
\hline$G F_{i 4}$ & Medium & Important \\
\hline$G F_{i 5}$ & Extreme good & Important \\
\hline
\end{tabular}

Table 3 Importance of subfeatures

\begin{tabular}{cc}
\hline Linguistic variable & Intuitionistic fuzzy numbers \\
\hline Very important & $(0.90,0.05,0.05)$ \\
\hline Important & $(0.75,0.20,0.05)$ \\
\hline Medium & $(0.50,0.40,0.10)$ \\
\hline Unimportant & $(0.25,0.60,0.15)$ \\
\hline Very unimportant & $(0.10,0.80,0.10)$ \\
\hline
\end{tabular}

Table 4 Weight value of each subfeature

\begin{tabular}{cc}
\hline Features & Weighting factor \\
\hline$G F_{i 1}$ & 0.22 \\
\hline$G F_{i 2}$ & 0.15 \\
\hline$G F_{i 3}$ & 0.26 \\
\hline$G F_{i 4}$ & 0.15 \\
\hline$G F_{i 5}$ & 0.22 \\
\hline
\end{tabular}

Table 5 Intuitionistic fuzzy number and feature value of each subfeature in correspondence

\begin{tabular}{cc}
\hline Features & Intuitionistic fuzzy numbers \\
\hline$G F_{i 1}$ & $(0.75,0.15,0.10)$ \\
\hline$G F_{i 2}$ & $(0.85,0.10,0.05)$ \\
\hline$G F_{i 3}$ & $(0.85,0.10,0.05)$ \\
\hline$G F_{i 4}$ & $(0.50,0.40,0.10)$ \\
\hline$G F_{i 5}$ & $(0.95,0.05,0.00)$ \\
\hline
\end{tabular}

$$
I F W A_{\omega}\left(\alpha_{1}, \alpha_{2}, \ldots, \alpha_{n}\right)=\omega 1 \alpha 1 \oplus \omega 2 \alpha 2 \oplus \ldots \oplus \omega n \alpha n
$$

Where, $\omega=(\omega 1, \omega 2, \ldots, \omega n)^{T}$ is the weight factor of, $\alpha_{j}(j=1,2$, $\ldots, n)$ and $\omega_{j} \in[0,1](j=1,2, \ldots, n), \sum_{j=1}^{n} \omega_{j}=1$

Weight calculation Equation:

$$
\lambda_{k}=\frac{\mu_{k}+\frac{\pi_{k} \mu_{k}}{\mu_{k}+v_{k}}}{\sum_{k=1}^{n}\left(\mu_{k}+\frac{\pi_{k} \mu_{k}}{\mu_{k}+v_{k}}\right)}
$$

Where, $\lambda_{k} \geq 0 ; k=1,2, \ldots, n ; \sum_{k=1}^{n} \lambda_{k}=1$.

As a matter of convenience, this thesis assumes that certain uncertain green feature is polymerized by five subfeatures- $G F_{i 1}, G F_{i 2}$, $G F i_{3}, G F_{i 4}, G F_{i 5}$, the value and importance of each are shown in Table 2.

Linguistic variables for importance of subfeatures and intuitionistic fuzzy numbers in correspondence as follows:

Assume that the importance degree of subfeature $G F_{k}$ is expressed by intuitionistic fuzzy number $D_{k}=\left(\mu_{k}, v_{k}, \pi_{k}\right)$. Then the weight of five subfeatures as in Table 4 can be obtained according to the corresponding relation in Table 3 and the weight calculation Eq. (16).

According to the correspondence in Table 4, the intuitionistic fuzzy number in correspondence with feature value of each subfeature can be 
figured out, just as presented in Table 5.

Using fuzzy algorithms (Eqs. (13) and (14)) and intuitionistic fuzzy weighted average operator (Eq. (15)), the intuitionistic fuzzy number can be calculated out as: $\alpha=(0.84,0.10)$

It can thus be seen that the green feature value of the component is 0.84 , which is very close to 'Very good' level.

Degree of uncertainty: $\pi_{\alpha}=1-0.84-0.10=0.06$.

\subsection{Processing of Quantitative Feature}

For quantitative uncertain green feature, average value and coefficient of variation can be figured out by using sample size statistics of same features within the industry, and then the standard deviation can be calculated, which lead us to obtain the value of the green feature and analyze the uncertainty of data by applying Monte Carlo simulated analysis. With regard to the probability distribution function in Monte Carlo simulation, the method of fitting analysis can be used to define the probability distribution function of statistics as it is hard to be gotten. Normal distribution, uniform distribution, Poisson distribution and t-distribution etc. are the commonly used probability distribution functions.

\section{Life Cycle Impact Assessment}

Life Cycle Impact Assessment (LCIA) aims to ascertain the impact that a production system gives the external environment (ecosystem, human health, resource consumption, etc.) for the material and energy exchange through characterization and evaluation of the material consumptions, the energy consumption and emissions data provided by inventory analysis. LCIA is comparatively mature after being researched by masses of scholars and organizations. There are many representative life cycle impact assessment methods internationally, which are usually divided into two categories: midpoint method and endpoint method. ${ }^{22}$ The former focuses on the category of environmental impact and its mechanism of action, which uses various characteristic factors to describe the relative importance of various environmental interference factors. While the latter focuses on the causality of environmental impact problems. Many researchers concluded that for the same inventory data, different impact evaluation results can be obtained by using different methods of impact assessment. ${ }^{23-25}$ At present, the mainstream of international life cycle impact assessment methods include CML2002, Eco-indicator99, EDIP2003, EPS 2000, Ecopionts2006, IMPACT 2002, LIME, etc.

The above methods respectively have different characteristics of their own; we can choose LCIA method according to the characteristics of the product evaluated and its main environmental impact types. With the LCIA method selected, the inventory data can be evaluated according to the steps of life cycle impact assessment. The steps of life cycle impact assessment are provided by ISO14044, GB 24040-2008 and GB 24040-2008.

\section{Examples of Verification}

According to the requirements of a ventilation system, the main index of the fan module $M_{0}$ is shown in the type of matter-element, as follows:

$$
R=\left[\begin{array}{ccc}
c_{1} & \text { Boost }(\mathrm{KPa}) & 15 \\
c_{2} & \text { Flow }\left(\mathrm{m}^{3} / \mathrm{min}\right) & 5 \\
c_{3} \text { Re volving } \operatorname{Speed}(\mathrm{r} / \mathrm{min}) & 2950 \\
c_{4} & \text { Shaft Power }(\mathrm{KW}) & 1.1 \\
c_{5} & \text { Weight }(\mathrm{Kg}) & 152
\end{array}\right]
$$

Five fan modules are selected to match the index above. According to the contents of 3.1 , the composite matrix is obtained as follows:

$$
R_{m n}=\left[\begin{array}{ccccccc} 
& M_{0} & M_{1} & M_{2} & M_{3} & M_{4} & M_{5} \\
C_{1} & 15 & 9.8 & 19.6 & 19.6 & 9.8 & 9.8 \\
C_{2} & 5 & 5.77 & 5.48 & 4.32 & 4.49 & 7.92 \\
C_{3} & 2950 & 2950 & 2950 & 1450 & 1450 & 1450 \\
C_{4} & 1.1 & 1.19 & 2.37 & 2.19 & 1.1 & 1.84 \\
C_{5} & 152 & 152 & 152 & 197 & 197 & 350
\end{array}\right]
$$

The composite element is normalized by Formula 3, as follows:

$$
R_{m n}^{\prime}=\left[\begin{array}{ccccccc} 
& M_{0} & M_{1} & M_{2} & M_{3} & M_{4} & M_{5} \\
C_{1} & 0.419 & 0.274 & 0.548 & 0.548 & 0.274 & 0.274 \\
C_{2} & 0.364 & 0.415 & 0.399 & 0.314 & 0.327 & 0.576 \\
C_{3} & 0.5182 & 0.518 & 0.518 & 0.255 & 0.255 & 0.255 \\
C_{4} & 0.262 & 0.283 & 0.564 & 0.522 & 0.262 & 0.438 \\
C_{5} & 0.293 & 0.293 & 0.293 & 0.380 & 0.380 & 0.674
\end{array}\right]
$$

The weight of each index is shown below according to its importance in the program,

$$
W=(0.3,0.4,0.1,0.1,0.1)
$$

Through Formula 4, Similarity of each module to the ideal module is got as follows:

$$
S=(0.086099,0.120778,0.142696,0.120721,0.221283)
$$

Where, $S_{1}<S_{4}<S_{2}<S_{3}<S_{5}$. So, the $M_{1}$ is the best match module. The following analysis will focus on the information of the module $M_{1}$.

Through data collection in some company, part of the design information of the fan is obtained, such as shown in Formula 20. And the material green feature $G F_{1}$ and manufacturing green feature $G F_{2}$ can be obtained according to Part 4 . Where, both gray iron and $45 \#$ steel are calculated as steel in the mapping of material green features. Besides, mapping of manufacturing green features requires a lot of database and knowledge base for support. Therefore, the green features are mainly obtained based on actual production experience of the company in this instance. And the water consumption, cutting fluids, noise, power consumption and dust are the value of the entire manufacturing process. With the limitation of data obtained, only raw material and manufacturing stages were taken into consideration. 


\begin{tabular}{|c|c|c|c|c|c|}
\hline Material & $\begin{array}{c}\text { Weight/ } \\
\text { Kg }\end{array}$ & $\begin{array}{c}\text { Manufac } \\
\text { turing }\end{array}$ & $\begin{array}{l}\text { Tolerance } \\
\text { Grade }\end{array}$ & \multicolumn{2}{|c|}{$\begin{array}{l}\text { Roughness } \\
\text { Ra }\end{array}$} \\
\hline \multirow{3}{*}{$H T 200$} & \multirow{4}{*}{25} & Milling & 9 & 6.3 & \\
\hline & & Boring & 8 & 3.2 & A \\
\hline & & Drilling & 9 & 6.3 & \\
\hline \multirow{3}{*}{$H T 250$} & & Milling & 9 & 6.3 & \\
\hline & \multirow[t]{2}{*}{15} & Boring & 8 & 3.2 & B \\
\hline & & Drilling & 9 & 6.3 & \\
\hline \multirow{3}{*}{$H T 250$} & \multirow{3}{*}{8} & Milling & 8 & 3.2 & \\
\hline & & Boring & 8 & 3.2 & C \\
\hline & & Planing & 9 & 6.3 & \\
\hline \multirow{3}{*}{$45^{\#}$} & \multirow{3}{*}{5} & Turning & 7 & 1.6 & \\
\hline & & Milling & 9 & 6.3 & $\mathrm{D}$ \\
\hline & & $\begin{array}{l}\text { Milling } \\
\text { Tempering }\end{array}$ & 9 & 3.2 & \\
\hline \multirow{3}{*}{$45^{\#}$} & \multirow{4}{*}{3.7} & Turning & 7 & 1.6 & \\
\hline & & Milling & 9 & 6.3 & $E$ \\
\hline & & $\begin{array}{l}\text { Milling } \\
\text { Tempering }\end{array}$ & 9 & 3.2 & \\
\hline \multirow{3}{*}{$H T 150$} & & Milling & 9 & 6.3 & \\
\hline & \multirow[t]{3}{*}{17} & Drilling & 9 & 6.3 & F \\
\hline & & Milling & 10 & 6.3 & \\
\hline \multirow{3}{*}{$H T 150$} & & Milling & 9 & 6.3 & \\
\hline & \multirow[t]{2}{*}{11} & Drilling & 9 & 6.3 & $G$ \\
\hline & & Milling & 10 & 6.3 & \\
\hline Parts & \multicolumn{3}{|c|}{$\begin{array}{c}\text { Assembling } \\
\text { Balance }\end{array}$} & & $\mathrm{H}$ \\
\hline
\end{tabular}

Green feature of raw materials stage:

$$
G F_{1}=\left[\begin{array}{cc}
\text { Material } & \text { Weight } / \mathrm{Kg} \\
\text { Steel } & 92.7
\end{array}\right]
$$

reen feature of manufacturing stage:

$$
G F_{1}=\left[\begin{array}{ccccc}
\text { Water } & \text { Cutting Fluid } & \text { Noise } & \text { Power } & \text { Dust } \\
10 \mathrm{Kg} & 0.4 \mathrm{Kg} & 85 \mathrm{~dB} & 572 \mathrm{Kwh} & 5 \mathrm{mg} / \mathrm{m}^{3}
\end{array}\right]
$$

Electrical energy consumption existed uncertain information in the statistical process. Power consumption and uncertainty of eight parts can be obtained respectively through statistical and estimation, as shown in Table 6.

The random value of each subfeature is obtained through Monte Carlo simulation with Matlab software, and the value of $\mathrm{GF}_{24}$ can be obtained through averaging. With calculating, power consumption is $582.6 \mathrm{Kwh}$.

Based on the extraction of green features, the main green data is available of the program, as shown in Table 7 :

According to data above, the program evaluation results $125 \mathrm{Pt}$ is got with Eco-indicator99 Evaluation Model.

\section{Conclusions}

A rapid life cycle assessment method for conceptual design based
Table 6 Uncertain green feature value and uncertainty

\begin{tabular}{ccc}
\hline Part & Power/Kwh & Uncertainty \\
\hline A & 46 & $\pm 10 \%$ \\
\hline B & 172 & $\pm 10 \%$ \\
\hline C & 138 & $\pm 10 \%$ \\
\hline D & 95 & $\pm 10 \%$ \\
\hline E & 88 & $\pm 10 \%$ \\
\hline F & 8 & $\pm 10 \%$ \\
\hline G & 14 & $\pm 10 \%$ \\
\hline H & 11 & $\pm 10 \%$
\end{tabular}

Table 7 Summarizes the blower green data

\begin{tabular}{cccc}
\hline Number & Type & Value & Unit \\
\hline$G F_{11}$ & Steel & 92.7 & $\mathrm{Kg}$ \\
\hline$G F_{21}$ & Water & 10 & $\mathrm{Kg}$ \\
\hline$G F_{22}$ & Cutting fluid & 0.4 & $\mathrm{Kg}$ \\
\hline$G F_{23}$ & Noise & 85 & $\mathrm{~dB}(\mathrm{~A})$ \\
\hline$G F_{24}$ & Power & 582.6 & $\mathrm{Kwh}$ \\
\hline$G F_{25}$ & Dust & 5 & $\mathrm{mg} / \mathrm{m}^{3}$ \\
\hline
\end{tabular}

on green feature was introduced in this paper. The RLCA requires obtaining the inventory data accurately and rapidly. And it mainly reflected the rapid in formation of product program, extracting and mapping of green features, uncertain information processing, concluded in the following:

Firstly, Green Feature is proposed in supporting the LCA in conceptual design stage, where a mapping relationship is established between green feature and design information to achieve the transformation from the design information to green features. So, the conventional design information can be translated to green features rapidly to provide relatively accurate inventory data for LCIA.

Secondly, product conceptual design model is proposed based on modular configuration. Product conceptual design will be done from the perspective of the whole life cycle and modular design, and the approximate products program will be obtained through retrieval and matching of each module of product using the knowledge base and case base. This would not only avoid the issues such as less information, high uncertainty etc. in conceptual design stage, but also get the necessary green information from the conception stage, which greatly improves the design efficiency and saves time and costs.

Thirdly, Intuitionistic Fuzzy theory and Monte Carlo method are respectively used to process the qualitative and quantitative uncertain information of green features in order to ensure the accuracy of the evaluation results. The uncertain information processing of uncertain green features can ensure the reliability of assessment result.

Consequently, the rapid life cycle assessment of the product design program is completed to support product green design.

\section{ACKNOWLEDGEMENT}

This work was financially supported by the National Natural Science Foundation of China (No. 51175312) and the National High Technology Research and Development Program (No. 2014AA041 503). 


\section{REFERENCES}

1. Ahn, S.-H., "An Evaluation of Green Manufacturing Technologies based on Research Databases," Int. J. Precis. Eng. Manuf.-Green Tech., Vol. 1, No. 1, pp. 5-9, 2014.

2. Zhang, X., "Simplified Method of Life Cycle Assessment and Its Case Study," M.Sc. Thesis, School of Electro-Mechanical Engineering, Guangdong University of Technology, 2013.

3. Ryu, J., Kim, I., Kwon, E., and Hur, T., "Simplified Life Cycle Assessment for Eco-Design," Proc. of the $3^{\text {rd }}$ IEEE International Symposium on Environmentally Conscious Design and Inverse Manufacturing, EcoDesign'03, pp. 459-463, 2003.

4. Graedcl, T. E., "Weighted Matrices as Product Life Cycle Assessment Tools," The International Journal of Life Cycle Assessment, Vol. 1, No. 2, pp. 85-89, 1996.

5. Hur, T., Lee, J., Ryu, J., and Kwon, E., "Simplified LCA and Matrix Methods in Identifying the Environmental Aspects of a Product System," Journal of Environmental Management, Vol. 75, No. 3, pp. 229-237, 2005.

6. Gao, Y., Liu, Z.-F., Huang, H.-H., and Li, B.-B., "The Research of Green Products Assessment Method based on Weighing Met Matrix," Machine Design \& Research, Vol. 24, No. 3, pp. 10-14, 2008.

7. Lee, H., Dornfeld, D. A., and Jeong, H., "Mathematical ModelBased Evaluation Methodology for Environmental Burden of Chemical Mechanical Planarization Process," Int. J. Precis. Eng. Manuf.-Green Tech., Vol. 1, No. 1, pp. 11-15, 2014.

8. Lee, J. Y., Kang, H. S., and Noh, S. D., "Simulation-Based Analysis for Sustainability of Manufacturing System," Int. J. Precis. Eng. Manuf., Vol. 13, No. 7, pp. 1221-1230, 2012.

9. Gaha, R., Yannou, B., and Benamara, A., "A New Eco-Design Approach on CAD Systems," Int. J. Precis. Eng. Manuf., Vol. 15, No. 7, pp. 1443-1451, 2014.

10. Hua, M. and Zhang, T., "Data Quality Assessment of Life Cycle Inventory Analysis," Research of Environmental Science, Vol. 16, No. 5, pp. 55-58, 2003.

11. Lloyd, S. M. and Ries, R., "Characterizing, Propagating, and Analyzing Uncertainty in Life-Cycle Assessment: A Survey of Quantitative Approaches,” Journal of Industrial Ecology, Vol. 11, No. 1, pp. 161-179, 2007.

12. Benetto, E., Dujet, C., and Rousseaux, P., "Integrating Fuzzy Multicriteria Analysis and Uncertainty Evaluation in Life Cycle Assessment," Environmental Modelling \& Software, Vol. 23, No. 12, pp. 1461-1467, 2008.

13. Lo, S.-C., Ma, H.-W., and Lo, S.-L., "Quantifying and Reducing Uncertainty in Life Cycle Assessment using the Bayesian Monte Carlo Method," Science of the Total Environment, Vol. 340, No. 1, pp. 23-33, 2005.
14. Wang, L., Li, F., Li, J., and Wang, X., "Sensitivity and Uncertainty Analysis of Life-Cycle Assessment based on Multivariate Regression Analysis," Proc. of the $5^{\text {th }}$ ICRM International Conference on Responsive Manufacturing-Green Manufacturing, pp. 184-191, 2010.

15. Zhu, L., "Uncertainty Analysis of the Inventory in LCA," M.Sc. Thesis, School of Mechanical and Automotive Engineering, Hefei University of Technology, 2012.

16. Chiu, M.-C. and Teng, L.-W., "Sustainable Product and Supply Chain Design Decisions under Uncertainties," Int. J. Precis. Eng. Manuf., Vol. 14, No. 11, pp. 1953-1960, 2013.

17. Li, P., "Theory and Method Research on Product Conceptual Design based on Function-Structure Model," Ph.D. Thesis, School of Mechanical Engineering, Shandong University, 2010.

18. Zhang, S., "Several Method for Fuzzy Multiple Attribute DecisionMaking with Applications," Ph.D. Thesis, School of Computer Science and Technology, Xidian University, 2012.

19. Atanassov, K. T., "Intuitionistic Fuzzy Sets," Fuzzy Sets and Systems, Vol. 20, No. 1, pp. 87-96, 1986.

20. Xu, Z., "Intuitionistic Fuzzy Aggregation Operators," IEEE Transactions on Fuzzy Systems, Vol. 15, No. 6, pp. 1179-1187, 2007.

21. Boran, F. E., Genç, S., Kurt, M., and Akay, D., "A Multi-Criteria Intuitionistic Fuzzy Group Decision Making for Supplier Selection with Topsis Method," Expert Systems with Applications, Vol. 36, No. 8, pp. 11363-11368, 2009.

22. Finnveden, G., Hauschild, M. Z., Ekvall, T., Guinee, J., Heijungs, R., et al., "Recent Developments in Life Cycle Assessment," Journal of Environmental Management, Vol. 91, No. 1, pp. 1-21, 2009.

23. Bovea, M. and Gallardo, A., "The Influence of Impact Assessment Methods on Materials Selection for Eco-Design," Materials \& Design, Vol. 27, No. 3, pp. 209-215, 2006.

24. Raluy, R., Serra, L., Uche, J., and Valero, A., "Life-Cycle Assessment of Desalination Technologies Integrated with Energy Production Systems,” Desalination, Vol. 167, pp. 445-458, 2004.

25. Seo, S., Aramaki, T., Hwang, Y., and Hanaki, K., "Environmental Impact of Solid Waste Treatment Methods in Korea," Journal of Environmental Engineering, Vol. 130, No. 1, pp. 81-89, 2004. 